\title{
Proteomics identifies differentially expressed proteins in neonatal murine thymus compared with adults
}

Xinze Cai ${ }^{1,2}$, Wenyue Huang ${ }^{3}$, Ying Qiao ${ }^{1}$, Yang Chen ${ }^{1}$, Shuyan Du' ${ }^{1}$, Dong Chen ${ }^{1}$, Shuang Yu ${ }^{1}$, Ruichao Che ${ }^{1}$ and Yi Jiang ${ }^{1,2,4^{*}}$

\begin{abstract}
Background: The thymus is an immune organ essential for life and plays a crucial role in the development of T cells. It undergoes a fetal to adult developmental maturation process occurring in mouse during the postnatal months. The molecular modifications underlying these ontogenic changes are essentially unknown. Here we used a differential proteomic-based technique (2D-Difference Gel Electrophoresis) coupled with matrix-assisted laser desorption/ionization-time of flight (MALDI-TOF) mass spectrometry to search for key proteins in the postnatal development of the thymus. Eight different BALB/c mice were used in the study: four mice aged of 1 day (neonatal) and four mice aged of 60 days (adult). Protein samples derived from thymus were labeled and run in 2D-PAGE (Two-Dimensional Polyacrylamide Gel Electrophoresis). One whole-thymus tissue from each mouse was run on gels and each gel containing a pooled sample of the eight mice was run in parallel. The pooled sample was set as the internal pool, containing equal amount of each protein extract used in the experiment. Gels were matched and compared with Difference In-gel Analysis software. Differential spots were picked, in-gel digested and peptide mass fingerprints were obtained.

Results: Among the differentially regulated proteins in neonatal thymus group, 111 proteins were identified by mass spectrometry, of which 95 proteins were up-regulated and 16 proteins were down-regulated. The identified proteins belong to several functional categories, including cell proliferation, cycle and apoptosis, transcription regulation, signal transduction, nucleotide processing, proteolysis and translation, protein folding, metabolism, oxidoreduction, cytoskeleton, immune response, and embryonic development. The major interaction networks comprised of cellular function and maintenance, cellular assembly and organization, and metabolism were also identified by STRING analysis.
\end{abstract}

Conclusions: The demonstrated molecular changes are relevant for understanding thymus development as well as neonatal immune function, and they provide the diagnostic disease markers. Further studies will be required to describe in detail the role of the identified proteins in thymus maturation and in the specific functions of neonatal thymus.

Keywords: DIGE, Thymus, Development, Proteomics, Immune

\footnotetext{
* Correspondence: caixinze@gmail.com

${ }^{1}$ Central Laboratory, First Affiliated Hospital of China Medical University,

Shenyang 110001, China

2Department of Immunology, College of Basic Medical Sciences, China

Medical University, Shenyang 110001, China

Full list of author information is available at the end of the article
} 


\section{Introduction}

The thymus is essentially an epithelial organ, containing many developing lymphocytes and playing a crucial role in the development of $\mathrm{T}$ cells. Histologically, the thymus can be broadly divided into two subcompartments, the cortex and the medulla, each of which contains distinct populations of thymic epithelial cells (TECs) and mesenchymal cells, endothelial cells and dendritic cells [1]. The microenvironment of the thymus can produce a diverse repertoire of peripheral $\mathrm{T}$ cells, and the correct patterning and organization of thymus stromal components are crucial for thymus function. Defects in thymus function can result in serious health consequences, including immunodeficiency or autoimmunity.

Thymus undergoes major homeostatic postnatal functional modifications and the underlying molecular mechanisms are essentially unknown. In recent years, much progress has been made in identifying the transcription factors and signaling pathways that play a role in thymus organogenesis and T cell development [2]. Although much of these molecular insights involved in development and immune reactions come from gene expression data analyzed by microarray technologies, they are unable to provide information concerning translational regulation of expression or post-translational modification [3].

Proteomic analysis of global changes in protein expressed in neonatal and adult murine organs provides a useful method for detecting proteins that play a role in the developing processes [4-6]. It is proposed that during the development of the thymus, various signals are present in neonatal thymus which differ from the adult thymus. The homeostasis of neonatal thymus microenvironment is critical for the metabolism and immune response [7]. Systematic analysis of thymus protein expression profiles including information about protein signatures, localization and their quantitative changes are thus useful to thymus development and maturation.

Recently the two-dimensional difference gel electrophoresis (2-D DIGE) technique with fluorescent dyes has allowed quantitative analysis of separated proteins with high sensitivity [8]. In this study, we performed a comparative proteomic analysis of differentially expressed proteins in the thymus of mice aged 1 day (neonates) and 60 days (adults), and the function analysis and the crosstalk of the proteins would be provided, aiming to search for key proteins in the postnatal development of the thymus.

\section{Results}

Identification of differentially expressed proteins in murine thymus using 2-DE

Neonatal (labeled with Cy3 or Cy5) and adult (labeled with $\mathrm{Cy} 3$ or Cy5) spots were normalized to an internal standard (labeled with $\mathrm{Cy} 2$ ) containing equal amount of each protein extract used in the experiment. Differences in the two stages of thymus development are reflected in the proteomic profiles of the thymus by DIGE technology. A representative gel image is demonstrated from each group (Figure 1). Protein spots on the gel were clear and the majority of spots located at the region of $\mathrm{pH} 4-8$ and relative molecular weight of 20-100 kDa. DeCyder 2-D difference analysis software was used to analyze and match 2-D images of the two groups. Overall, the average number of protein spots was 2274, and approximately 1406 proteins were matched between the two groups. Among them, we detected 317 spots with an increased level in the neonatal thymus and 194 spots with a higher representation in the adult thymus. 111 proteins of interest with the difference over 1.5 -fold were identified by MALDI-TOF MS (Additional file 1: Table S1) and partial lists of them are indicated (Table 1).

\section{Ontogenic classification}

The identified proteins with significant differential displays between the neonatal and the adult thymus group were clustered into categories according to their biological function and subcellular localization (Figure 2). Functional classification of identified proteins were grouped into 11 categories, including cell proliferation, cycle and apoptosis (8\%), transcription regulation (4\%), signal transduction (6\%), nucleotide processing (13\%), proteolysis and translation (4\%), protein folding (12\%), metabolism (10\%), oxidoreduction (12\%), cytoskeleton (15\%), immune response (8\%), and embryonic development (8\%). According to the subcellular location, these proteins were classified into eight categories, including membrane (12\%), endoplasmic reticulum (8\%), mitochondrion (24\%), nucleus (10\%), cytoplasm (38\%), extracellular matrix and secreted (4\%), peroxisome $(2 \%)$ and undefined (2\%).

\section{Protein data mining}

The web-tool STRING is a database and web resource integrating information from numerous sources, including both physical and functional interactions [9]. Nodes represent the proteins and lines with different colors between nodes indicate different protein-protein interaction modes. Each interaction between nodes is supported from literatures. In this study, STRING identified the major networks comprised of cellular function and maintenance, cellular assembly, organization and metabolism, and it also showed the major interactions between the proteins which were differentially expressed (Figure 3).

\section{Validation of the differential protein displays by real time PCR and Western blotting}

In order to confirm the changes described, experiments were next performed to validate the proteins differently 


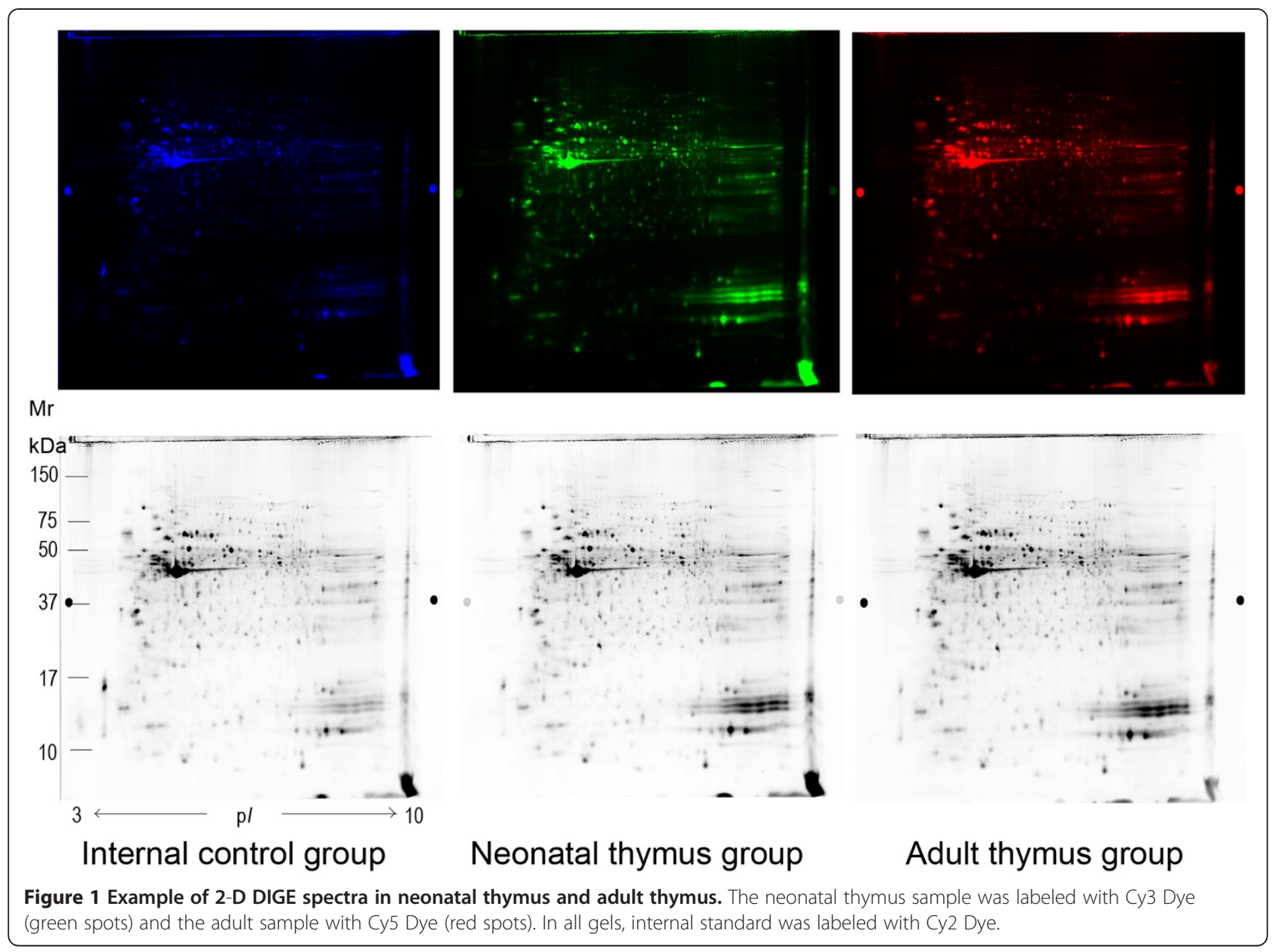

display between the neonatal thymus and the adult. We selected 12 proteins differentially regulated at the two stages and quantified their mRNA expression by quantitative RT-PCR. As shown in Table 2, quantitative RTPCR identified similar levels of mRNA regulation for these genes, indicating transcriptionally regulated expression of these proteins. We also analyzed samples by Western blotting using specific antibodies, which were directed against ACTN4, CH60, ACTB and COF1. The results obtained from Western blot were compatible with the intensities of the corresponding spots observed in 2-D gels (Figure 4). The results suggest that a proteomic differential display model is applicable to comparing.

\section{Discussion}

Proteomics studies, including 2-DE, MS, and bioinformatics tools, facilitate the direct understanding of the mechanism of the physiological or pathological process. A major critical issue of 2-DE involves reproducibility problems, owing to gel-to-gel and operatorto-operator variations [10,11]. To eliminate these technical limitations, investigators propose a complete workflow for DIGE image analysis and comparison in which cut-off values are carefully determined before extracting spots of interest according to the experimental conditions and reproducibility of the images analyzed. A detailed statistical analysis is also proposed on the identified spots using standard statistical tests $[4,12]$.

Comparative proteomic analysis of proteins in mammalian organ at one point of time may accelerate preand clinical development of more specific diagnostic and prognostic disease markers and new, more selective therapeutic interventions [3-5,13]. Our study compared proteins in thymus from mice aged 1 day (neonates) with mice aged 60 days (adults) to gain insights into the proteins involved in the postnatal development of the thymus and the immune system. We found that there were many differences in the proteins expressed in adult and neonatal thymus, with approximately 511 differentially expressed proteins by more than 1.5 -fold. Among them, 111 proteins were identified by MALDI-TOF MS. These proteins participate in the cell proliferation, cycle and apoptosis, transcription regulation, signal transduction, nucleotide processing, proteolysis and translation, 
Table 1 Partial lists of differentially regulated proteins in neonatal thymus group identified by MS*

\begin{tabular}{|c|c|c|c|c|c|c|c|c|}
\hline Accession No. & Protein name & $\begin{array}{l}\text { Gene } \\
\text { name }\end{array}$ & $\mathrm{pl} / \mathrm{MW}$ & $\begin{array}{c}\text { Mass } \\
\text { matched }\end{array}$ & Protein covered (\%) & $\begin{array}{l}\text { Mascot } \\
\text { score }\end{array}$ & $\begin{array}{l}t \text {-test } \\
\text { value }\end{array}$ & $\begin{array}{c}\text { Fold } \\
\text { change }\end{array}$ \\
\hline TENA_MOUSE & Tenascin & Tnc & $4.77 / 237$ & $11 / 22$ & 8 & 70 & 0.00019 & $2.03 \uparrow$ \\
\hline ACTN4_MOUSE & Actinin-4 & Actn4 & $5.25 / 105$ & $16 / 42$ & 21 & 93 & 0.000054 & $1.74 \uparrow$ \\
\hline TRAP1_MOUSE & Heat shock protein $75 \mathrm{kDa}$ & Trap1 & $6.25 / 81$ & $22 / 35$ & 35 & 212 & 0.0000037 & $1.54 \uparrow$ \\
\hline GRP75_MOUSE & Stress-70 protein & Hspa9 & $5.91 / 74$ & $19 / 29$ & 29 & 166 & 0.0000022 & $1.65 \uparrow$ \\
\hline CH60_MOUSE & $60 \mathrm{kDa}$ heat shock protein & Hspd1 & $5.91 / 61$ & $13 / 25$ & 32 & 129 & 0.00012 & $1.90 \uparrow$ \\
\hline ATPB_MOUSE & ATP synthase subunit beta & Atp5b & $5.19 / 56$ & $21 / 24$ & 58 & 253 & 0.0000023 & $2.45 \uparrow$ \\
\hline SEPT7_MOUSE & Septin-7 & Sept7 & $8.73 / 51$ & $4 / 16$ & 12 & 103 & 0.000071 & $2.72 \uparrow$ \\
\hline ACTB_MOUSE & Beta-actin & Actb & $5.29 / 42$ & $15 / 45$ & 42 & 109 & 0.000075 & $2.20 \uparrow$ \\
\hline STML2_MOUSE & Stomatin-like protein 2 & Stoml2 & $8.95 / 38$ & $14 / 24$ & 45 & 165 & 0.00000052 & $2.70 \uparrow$ \\
\hline PSME1_MOUSE & $\begin{array}{l}\text { Proteasome activator } \\
\text { complex subunit } 1\end{array}$ & Psme1 & $5.73 / 29$ & $13 / 28$ & 45 & 123 & 0.000015 & $3.57 \downarrow$ \\
\hline COF1_MOUSE & Cofilin-1 & $\mathrm{Cfl} 1$ & $8.22 / 19$ & $7 / 9$ & 34 & 96 & 0.00000032 & $9.36 \downarrow$ \\
\hline COTL1_MOUSE & Coactosin-like protein & Cotl1 & $5.28 / 16$ & $14 / 29$ & 76 & 213 & 0.0000072 & $2.28 \downarrow$ \\
\hline
\end{tabular}

"CyDye images were analyzed by BVA and spots that showed statistically significant differences $(p<0.05)$ in intensity between the neonatal and the adult thymus groups are listed. pl: calculated isoelectric point; MW: nominal molecular weight; $\uparrow$ : up-regulated; $\downarrow$ : down-regulated.

protein folding, metabolism, oxidoreduction, cytoskeleton, immune response, and embryonic development.

According to our data, the proteins involved in structuring actin cytoskeleton have emerged from this study. Actb (Beta-actin), Actn4 (Actinin-4) and Sept7 (Septin-7) were found more expressed in the neonatal thymus compared to adult; Cfl1 (Cofilin-1) and Cotl1 (Coactosin-like protein) were found less expressed in the neonatal thymus. These proteins play a role in regulating the actin cytoskeleton, including filament polymerization and depolymerization. Increasing knowledge shows that the dynamic actin cytoskeleton, consisting of actin isoforms and their binding proteins, is essential for all developmental processes and the viability of the adult organism [14]. These functions are attributed to the ability of actin to form filaments that can rapidly assemble and disassemble according to the needs of the cell [15].

Actb is a highly conserved actin isoform ubiquitously expressed in vertebrates and mice hypomorphic for Actb die of uncharacterized defects during development $[16,17]$. Actn4 is an actin-binding protein and has been reported to crosslink actin, regulate actin cytoskeleton and enhance cell mobility [18]. Sept7, another cytoskeletal component, can assemble on the T-cell cortex and be enriched in filaments for efficient motion of motile $\mathrm{T}$ cells [19]. It is demonstrated that actin cytoskeleton and
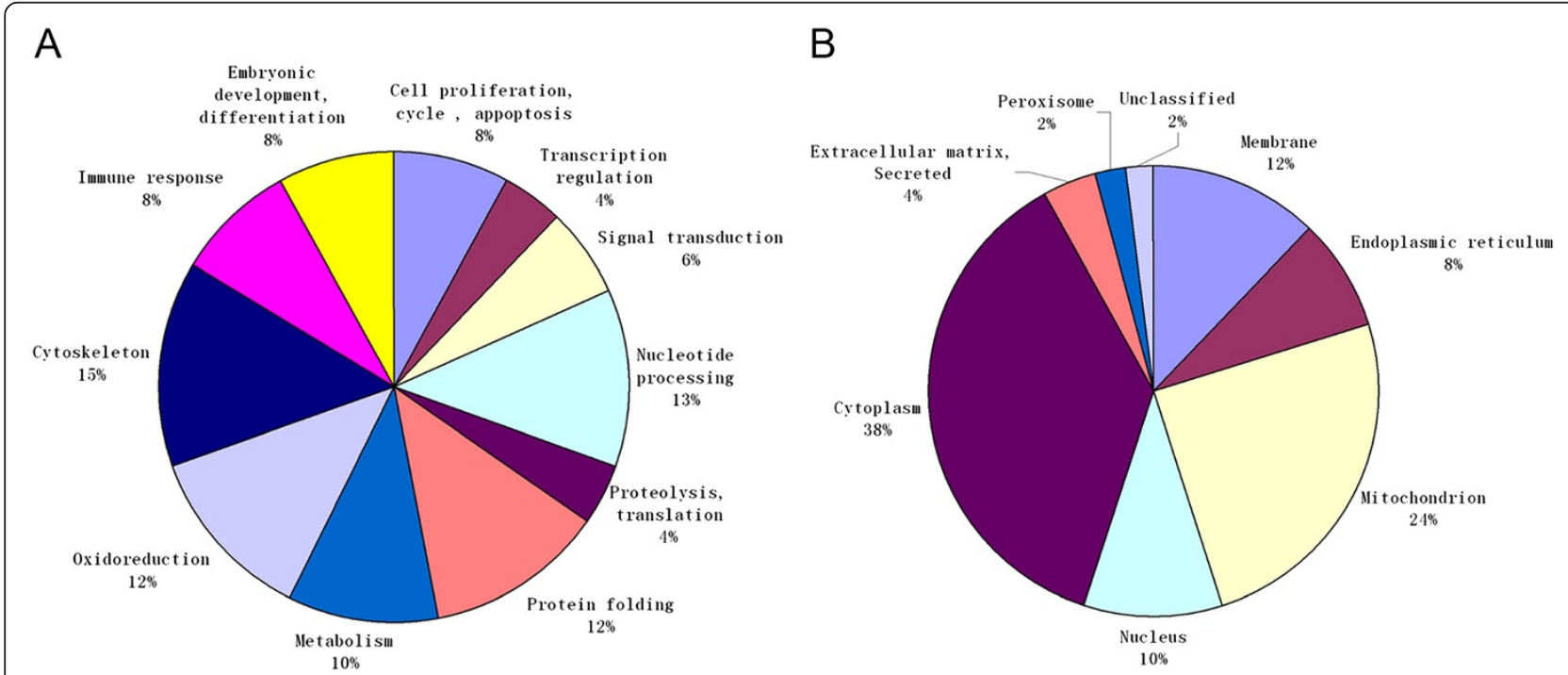

Figure 2 Classification of proteins by gene ontology according to their biological function (A) and subcellular localization (B).

Assignments were made on the basis of information provided from the Swiss-Prot database. 


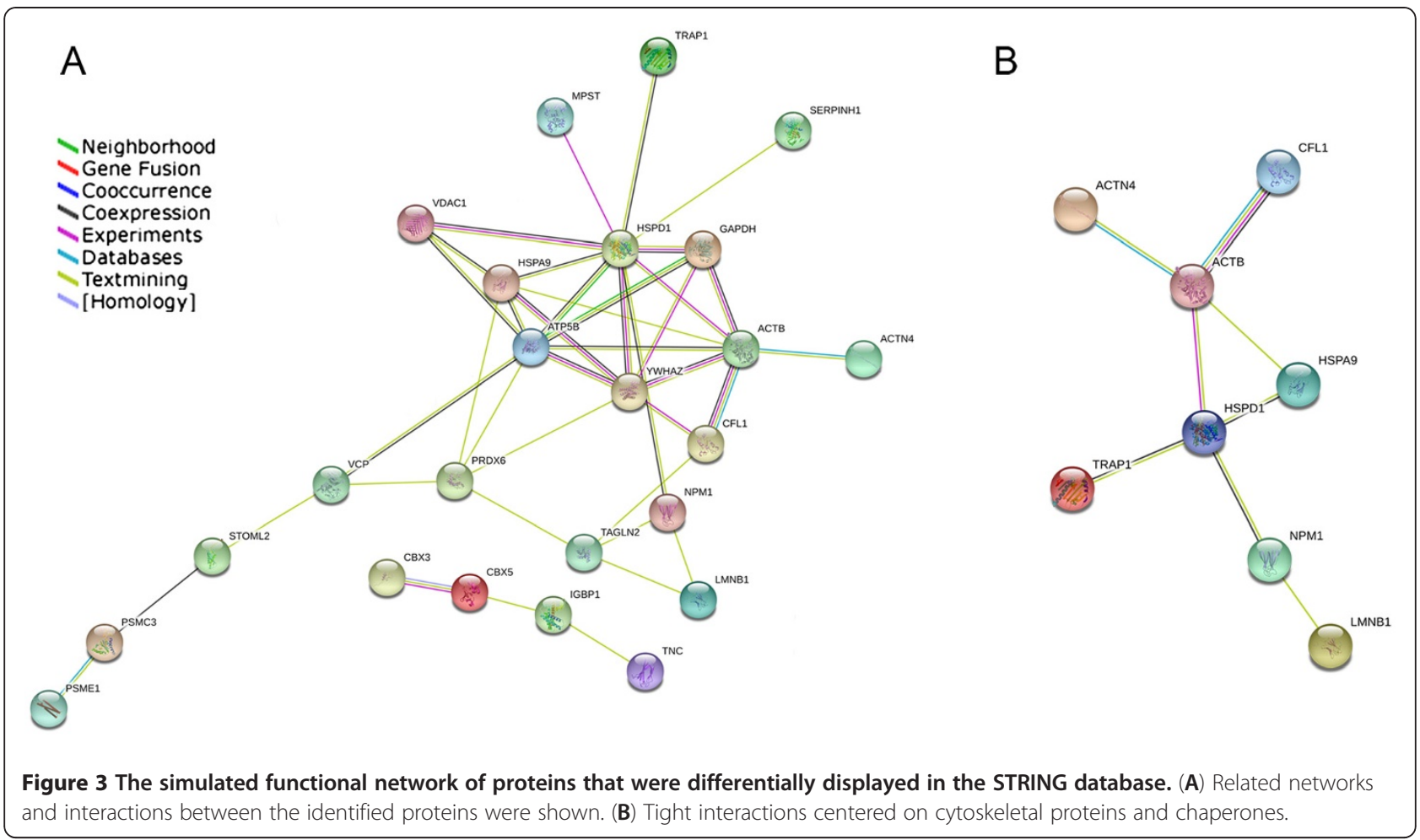

TCR signaling complexes are tightly integrated in T cells. Various cytoskeletal elements are crucial for the finetuning of $\mathrm{T}$ cell signaling and the immunological synapse (IS), while $\mathrm{T}$ cell activation induces the organization of both microtubules and actin cytoskeleton [20,21]. Thus these up-regulated cytoskeletal proteins assemble and contribute to T-cell activation and the IS formation in neonatal murine thymus. Additionally, we found Cfl1 and Cotl1 were down-regulated in the neonatal mice. It is reported that decreased Cfl1 expression is important for early mouse embryo development, and Cotl1 is associated with autoimmune disorders [22-24]. Both proteins belong to the actin depolymerizaition factor family and interact with actins and filaments to function primarily in promoting depolymerization [24,25]. Therefore, homeostasis of actin dynamics is important for the postnatal development of mice thymus.

The mitochondrial protein Stoml2 (Stomatin-like protein 2) was found more expressed in neonatal mice. It has been suggested that Stoml2 expression is dramatically up-regulated during $\mathrm{T}$ cell activation, and this increases $\mathrm{T}$ cell function and mitochondrial biogenesis, ultimately leading to resistance to apoptosis [26]. Interestingly, it is reported that Stoml2 is also involved in the organization of the peripheral cytoskeleton and acts as a functional bridge between TCR signalosomes and the cytoskeleton and cellular organelles [27].

These differentially expressed proteins involved in cytoskeleton household in neonatal compared to adult thymus are crucial for sustaining $\mathrm{T}$ cell activation and regulating cytoskeleton rearrangements. The regulation of cytoskeleton reorganization by $\mathrm{T}$ cell activation, and conversely, the control of $\mathrm{T}$ cell activation by cytoskeleton can be areas of active investigation. The disordered expression of these cytoskeleton proteins in neonatal thymus may be responsible for immune system diseases. Physiological variations occurring in the course of thymus maturation is a mandatory step to delineate the pathological mechanisms for the immune diseases which

Table 2 Differences in mRNA expression levels of the proteins differentially expressed in neonatal versus adult thymus

\begin{tabular}{lc}
\hline Gene name & mRNA ratio (Neonate/Adult) \\
\hline Thc & 1.42 \\
Atp5b & 1.63 \\
Actn4 & 1.78 \\
Actb & 1.71 \\
Hspd1 & 1.48 \\
Hspa9 & 2.23 \\
Trap1 & 1.55 \\
Sept7 & 1.89 \\
Stoml2 & 1.60 \\
Cfl1 & 0.76 \\
Cotl1 & 0.68 \\
Psme1 & 0.61 \\
\hline
\end{tabular}




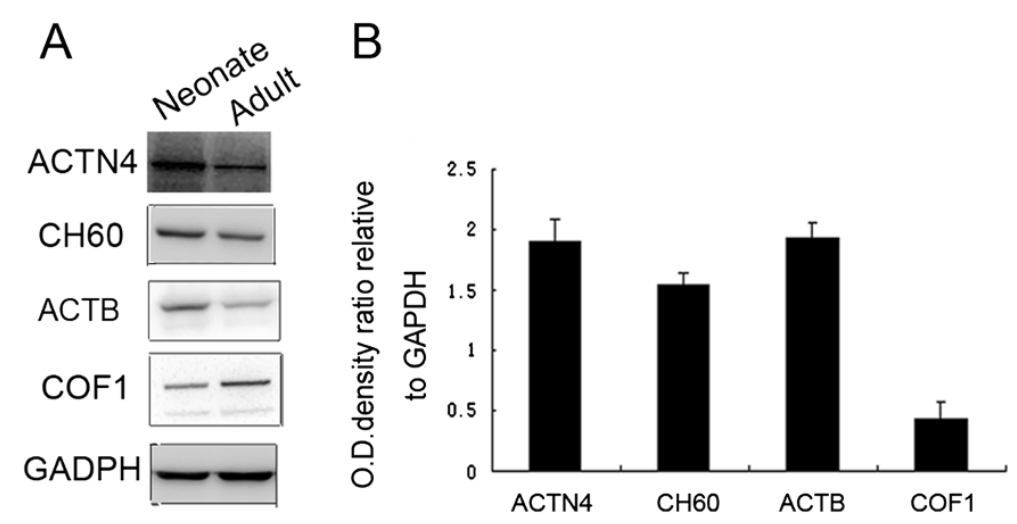

Figure 4 Validation of differentially displayed proteins between the neonatal and adult thymus. (A) $60 \mu \mathrm{g}$ of protein extracts was used for protein detection. There were more abundant ACTN4, CH60 and ACTB expression, while less COF1 expression in neonates. GADPH was used as a normalization control. (B) A summary of densitometric analysis of Western blotting was shown as mean \pm SE calculated from three experiments. The results were normalized to individual GAPDH expression.

is related to cytoskeleton disruption. Accordingly, the cytoskeleton and its proteins greatly contribute to thymus maturation in neonatal mice, and cytoskeletal proteins can be potential targets for immunomodulation. Dysfunction of the cytoskeleton proteins in the development of thymus could lead to serious health consequences, including immunodeficiency or autoimmunity. Further investigation will be needed, and gene knockout or small molecular inhibitors that target these genes may be useful for understanding their effects in immune development.

Among the up-regulated proteins in neonatal mice, we also identified some heat shock proteins (HSPs) including Hspd1 (60 kDa heat shock protein), Trap1 (Heat shock protein $75 \mathrm{kDa}$ ) and Hspa9 (Stress-70 protein). These proteins represent a set of highly conserved molecular chaperones that serve by folding newly synthesized proteins, disassembling unstable proteins, and assisting in the transportation of proteins within the cell [28]. They enable cells to survive adverse environmental conditions, and their absence damages the embryonic development in mice [29,30]. Christensen et al. show that homozygosity for the null allele of Hspd1 causes early embryonic lethality, while heterozygosity for the inactivated allele permits embryonic development and postnatal survival [30]. It is worth noting that eukaryotic and prokaryotic HSPs have high sequence homology and HSPs could act as potentially dangerous autoantigens, which adds to the evidence that neonatal mice are more susceptible to autoimmune disease than adult mice [31]. In addition, Npm1 (Nucleophosmin) was found highly expressed in neonatal thymus. Npm1 is a nuclear chaperone involved in chromatin remodeling during embryonic development and plays important roles in the regulation of cell proliferation and anti-apoptosis [32,33]. Loss of Npm1 impairs embryonic development and leads to premature cellular senescence and genomic instability [34]. Regarding these up-regulated chaperones above identified in neonatal thymus, it indicates their important roles during neonatal period.

Besides the proteins related with cytoskeleton and chaperones, other differentially expressed proteins were also identified. For instance, Tnc (Tenascin) is a glycoprotein of the extracellular matrix, which is involved in lymphocyte differentiation and migration. It is demonstrated that Tnc is expressed by epithelial cells early during embryonic development of the thymus [35,36]. It can support the tethering and rolling of lymphocytes, which would be used by lymphocytes migrating through secondary lymphoid organs [37]. Newborn mice are considered lymphopenic and the number of cells in the periphery is gradually increased by the constant output of newly exported $\mathrm{T}$ cells from the thymus $[38,39]$. Tnc is up-regulated in neonatal murine thymus, suggesting its important role in lymphocytic migration.

In addition, we also identified some proteins upregulated in adult thymus. For example, Psme1 (Proteasome activator complex subunit 1) is implicated in immunoproteasome assembly and required for efficient antigen processing. Immune proteasomes in thymus are involved in processing of self-antigen, which are presented by MHC class I molecules for rejection of autoreactive thymocytes in adults $[40,41]$. They are present in adult thymus and responsible for negative selection of thymocytes through apoptosis. It is demonstrated that dexamethasone-induced thymocyte apoptosis is mediated by proteasomes, and lactacystin can also regulate apoptotic signaling as a proteasome-specific peptide inhibitor in the process of thymocyte apoptosis [42]. These implicate that such chemicals via regulating proteasome could be employed during the development, maturation or involution of thymus. 
Bioinformatics analysis was performed to classify identified proteins in neonates based on biological function and subcellular localization. It links the identified proteins to protein folding, metabolism, oxidoreduction, cytoskeleton, immune response, embryonic development and so on. Some findings reported that neonates exhibited aberrant immune responses when compared to adults, resulting in increased susceptibility to infection and autoimmune disease $[31,43]$. Our results indicated the associated categories of proteins possibly involved in immaturity of neonatal for immune development and their potential role in neonates.

Recently the functional connectivity within a proteome becomes more and more important. As various protein complexes, transient interactions and functional pathways are all context-dependent, we further investigated the interaction networks between these proteins by the STRING web-tool. Although it represents the union of all possible protein-protein links, STRING imports protein association knowledge not only from databases of physical interactions, but also from databases of curated biological pathway knowledge [9]. The potential gene and protein interactions indicated in the study may enable prioritization of genes of interest. STRING results shows that those cytoskeletal proteins and chaperones are parts of the network that links the differentially expressed proteins. Most identified proteins are connected by the two kinds of proteins. Observing their own interaction network, we also found that they clustered in a tight interaction network centered on ACTB, HSPA9 and HSPD1. It is known that the cytoskeleton is incomplete without its associated proteins, which include chaperones that appear to protect the cytoskeleton in circumstances where cytoskeletal homeostasis is affected. The interplay between the chaperone and actin cytoskeleton also indicates that chaperones are not only limited to solve abnormal situations, but they also have an active participation during the normal differentiation process of the cell and are key factors for structural and functional rearrangements. For example, it has been reported that Prefoldin, a hexameric chaperone which facilitates posttranslational folding of actins and other cytoskeletal proteins, is required for lymphocyte development and function [44]. Deficiency in Prefoldin would cause lymphopoiesis defects, including dramatic reductions in immature $\mathrm{CD}^{+} \mathrm{CD}^{+}$double-positive $\mathrm{T}$ cells in thymus, and the phenotype was consistent with an actin-folding defect. Therefore, chaperones interact closely with the cytoskeleton network in the process of thymus maturation, and the additional experiments will be needed to identify more protein members and clarify the interaction and function.

In conclusion, we have identified unique differentially displayed proteins focusing on a comparison of immune related proteomes between neonatal thymus and adult thymus. The demonstrated molecular changes are relevant for understanding thymus development as well as neonatal immune function. Further studies will be required to describe in detail the role of the identified proteins in thymus maturation and in the specific functions of neonatal thymus.

\section{Materials and methods \\ Animals}

Eight different BALB/c mice were used in the study: four mice aged of 1 day and four mice aged of 60 days. They were kept under 12:12 h cycle of light with ad libitum access to food and drink. Mice were killed in accordance with Institutional Animal Care and Use Committee of China Medical University guidelines and thymuses were quickly dissected and frozen under liquid nitrogen. One whole-thymus tissue from each mouse was run on gels and each gel containing a pooled sample of the eight mice was run in parallel. The pooled sample was set as the internal pool, containing equal amount of each protein extract used in the experiment.

\section{Sample preparation and labelling}

For protein solubilization, $1 \mathrm{mg}$ lyophilized thymus was suspended in $400 \mu \mathrm{l}$ rehydration buffer, i.e., $8 \mathrm{M}$ urea, $2 \mathrm{M}$ thiourea, $40 \mathrm{mM}$ Tris, 4\% CHAPS, $65 \mathrm{mM}$ DTT, $2 \%$ IPG buffer and 1\% protease inhibitor cocktail. The protein extracts were prepared for 2-DE by using 2-D clean-up kit (GE Healthcare) following manufacturer instructions. Precipitated proteins were resuspended in rehydration buffer and finally quantified using 2-D Quant Kit (GE Healthcare). The $\mathrm{pH}$ was adjusted to 8.5 by $100 \mathrm{mM} \mathrm{NaOH}$ and $50 \mu \mathrm{g}$ of protein in either group was labeled with 400 pmol of either Cy3 or Cy5 dyes (GE Healthcare). A $50 \mu \mathrm{g}$ protein mix, containing equal amount of each protein extract was labeled with 400 pmol of Cy2 dye as the internal standard sample. Labeled samples were immediately subjected to IPG strips (24 cm, pH 3-10, NL) and $900 \mu \mathrm{g}$ of total protein was mixed in the rehydration buffer for preparative isoelectric focusing (IEF).

\section{2-DE}

IEF was performed using a step-wise voltage ramp by IPGphor IIIsystem (GE Healthcare): $30 \mathrm{~V}$ for $12 \mathrm{~h}$, $300 \mathrm{~V}$ for $3 \mathrm{~h}$, linear ramping from $300 \mathrm{~V}$ to $1,000 \mathrm{~V}$ for $6 \mathrm{~h}$ and from $1,000 \mathrm{~V}$ to $8,000 \mathrm{~V}$ for $3 \mathrm{~h}$, and finally 8,000 $\mathrm{V}$ for $7 \mathrm{~h}$. Once IEF was completed, the strips were equilibrated in equilibration buffer (75 mM Tris- $\mathrm{HCl}, \mathrm{pH}$ 8.8, $6 \mathrm{M}$ urea, 30\% glycerol, $2 \%$ SDS and 1\% DTT) for $15 \mathrm{~min}$, followed by the same buffer containing $2.5 \%$ iodoacetamide instead of DTT for another $15 \mathrm{~min}$. The second dimension was performed using 12.5\% 
SDS-PAGE gel $\left(260 \times 200 \times 1 \mathrm{~mm}^{3}\right)$ at $1 \mathrm{~W}$ constant power per gel by Ettan DALTsix (GE Healthcare).

\section{Image analysis}

The gel was placed in the Typhoon 9400 Multi Scanner (GE Healthcare). Cy2, Cy3 and Cy5 fluorescencelabeled images were scanned at 488/520,532/580 and $633 / 670 \mathrm{~nm}$ wavelength pairs, respectively. Quantitative differential expression analysis was performed by DeCyder 6.5 sftware (GE Healthcare). Scanned images of fluorescenty labeled proteins were sequentially analyzed by differential in-gel analysis (DIA-module) during which the $\mathrm{Cy} 5: \mathrm{Cy} 2$ and $\mathrm{Cy} 3: \mathrm{Cy} 2$ normalization of protein spot was performed. The Log abundance ratios of each protein spot were then compared between neonatal and adult thymus from all gels by Biological Variation Analysis (BVA-module). Due to an intrinsic variability associated to the mouse peculiarities, we chose a stringent criterion: (i) a change of expression of at least 1.5 -fold, (ii) $t$-test value $(p<0.05)$, and (iii) the identification of the spots in the four experimental replicates.

\section{Mass spectrometry identification and bioinformatics analysis}

The differentially expressed protein spots were cut to reduction, alkylation, digestion, extraction, spot targeting and desalting. The sample plate was placed into MALDI-TOF mass spectrometer (Bruker Daltonics) for mass spectrometry (MS) analysis to obtain peptide mass fingerprinting (PMF). MS spectra were analyzed using the software flexAnalysis version 3.0 (Bruker Daltonics). Protein identification of peptide fragments was performed using MASCOT software (http://www. matrixscience.com) against Swiss-Prot database (Swiss Institute of Bioinformatics). Carbamidomethylation for cysteine, oxidation for methionine and other variants were also taken into consideration. Probability based on Mowse score $>58$ suggests a significant match and accurate identification of the protein.

\section{Interaction network}

Functional partnerships between proteins are the fundamental of cell working. A proteome-scale interaction network of the differentially expressed proteins that identified in the present search was derived from the STRING database (http://string-db.org) [9].

\section{Quantitative real-time RT-PCR}

Isolation of total RNA was carried out with the TRIzol (Invitrogen) according to the manufacturer's protocol. One microgram of total RNA was reverse transcribed to cDNA in a total volume of $20 \mu \mathrm{l}$ system using a RT reaction kit (Promega). Real-time PCR was performed using the Express SYBR greener qPCR supermix Universal Kit
(Invitrogen) on a Rotor-gene 6000 system (QIAGEN). The 25- $\mu$ l PCR mixture contained $2 \mu$ l reversetranscribed product $12.5 \mu \mathrm{l}$ SYBR Green supermix, $8.5 \mu \mathrm{l}$ RNase-free water, $1 \mu \mathrm{l}$ forward, and $1 \mu$ l reverse primers (Table 3 ). The reaction was incubated in a 72 -well optical plate by 45 amplification cycles of $94^{\circ} \mathrm{C}$ for $5 \mathrm{~s}, 58^{\circ} \mathrm{C}$ for $20 \mathrm{~s}$, and $72^{\circ} \mathrm{C} 30 \mathrm{~s}$. Each sample was analyzed in triplicate and repeated three times. Gene expression levels were calculated relative to the housekeeping gene Gapdh.

\section{Western blot}

To determine the expression of protein, tissue extracts were prepared from $1 \times 10^{6}$ cells in lysis buffer $(20 \mathrm{mM}$ Tris pH7.4, $250 \mathrm{mM}$ sodium chloride, 0.1\%TritonX-100, $2 \mathrm{mM}$ EDTA, $10 \mu \mathrm{g} / \mathrm{ml}$ leupeptin, $10 \mu \mathrm{g} / \mathrm{ml}$ aprotinin, $0.5 \mathrm{mM}$ phenylmethylsulfonyl fluoride, $4 \mathrm{mM}$ sodium orthovanadate and $1 \mathrm{mM} \mathrm{DTT}$ ), and $60 \mu \mathrm{g}$ of the protein was resolved on 12\% SDS-polyacrylamide gels. After electrophoresis, the proteins were eletrotransferred to nitrocellulose filters, the membrane (Amersham) was blocked with 5\% nonfat dry milk in TBS-T (20 mM Tris, $\mathrm{pH}$ 7.6, $137 \mathrm{mM} \mathrm{NaCl}, 0.05 \%$ Tween-20) for $3 \mathrm{~h}$ at room temperature, and the proteins were probed with specific antibodies-ACTN4, CH60, ACTB and COF1 (Cell Signaling) and detected by chemiluminescence

Table 3 Primers used for RT-PCR

\begin{tabular}{|c|c|c|}
\hline Gene name & Primer sequence $\left(5^{\prime}-3^{\prime}\right)$ & Amplicon size (bp) \\
\hline $\mathrm{Tnc}$ & $\begin{array}{l}\text { fwd: AGCCACCCGCTACTACAT } \\
\text { rev: CTGCACCTGAACGACAAA }\end{array}$ & 193 \\
\hline Atp5b & $\begin{array}{l}\text { fwd: AGATTCTGGTGACTGGGATA } \\
\text { rev: TGGCGACATTGTTGATTAG }\end{array}$ & 132 \\
\hline Actn4 & $\begin{array}{l}\text { fwd: GTTGCCTAAGCCAGAGC } \\
\text { rev: ATCATTCCCAGGGTCATC }\end{array}$ & 154 \\
\hline Actb & $\begin{array}{l}\text { fwd: ATCGTGCGTGACATCAAA } \\
\text { rev: AGAAGGAAGGCTGGAAAA }\end{array}$ & 178 \\
\hline Hspd1 & $\begin{array}{l}\text { fwd: GGGGAAGTCCCAAAGTAA } \\
\text { rev: CCTTGGCAATAGATCGTG }\end{array}$ & 174 \\
\hline Hspa9 & $\begin{array}{l}\text { fwd: CAAAGGTCCTGGAGAATG } \\
\text { rev: CAATAAGACGCTTAGTAGCA }\end{array}$ & 150 \\
\hline Trap1 & $\begin{array}{l}\text { fwd: AGACGGACGCACCACTCA } \\
\text { rev: CAGCCACTTGGGCAGGAT }\end{array}$ & 158 \\
\hline Sept7 & $\begin{array}{l}\text { fwd: GTGAATCTGGACTGGGAAAG } \\
\text { rev: CAGCAGCAACTGAACACCAC }\end{array}$ & 158 \\
\hline Stoml2 & $\begin{array}{l}\text { fwd: GGGCTCTGACTCAACATAAT } \\
\text { rev: GATTGGAGGGCAGTAGCA }\end{array}$ & 117 \\
\hline $\mathrm{Cfl} 1$ & $\begin{array}{l}\text { fwd: TGCCGCTATGCACTCTAT } \\
\text { rev: GGTCCTTGACCTCCTCGT }\end{array}$ & 199 \\
\hline Cotl1 & $\begin{array}{l}\text { fwd: AAGTTGGCCCTCATCACA } \\
\text { rev: ACTGAGCGTCGTAGTTGG }\end{array}$ & 199 \\
\hline Psme1 & $\begin{array}{l}\text { fwd: AGGAGGAGCGGAAGAAGC } \\
\text { rev: AACCAGGTAGTGACCAGATTGA }\end{array}$ & 184 \\
\hline Gapdh & $\begin{array}{l}\text { fwd: CCTTCCGTGTTCCTACCC } \\
\text { rev: AAGTCGCAGGAGACAACC }\end{array}$ & 163 \\
\hline
\end{tabular}


(Amersham). To assaure equal loading, gels were stripped and reprobed with antibodies against GAPDH (Shanghai Kangchen).

\section{Additional file}

Additional file 1: Table S1. Lists of differentially regulated proteins in neonatal thymus group identified by $\mathrm{MS}^{*}$.

\section{Competing interests}

The authors declare that they have no competing interests.

\section{Authors' contributions}

XC carried out experiments and data analysis, and composed the draft. WH carried out data acquisition and interpretation. YQ, YC and SD contributed to the proteomic analysis. SY and RC participated in supervision of the study. YJ contributed to the project idea and obtained grant funding. All authors have read and approved the final manuscript.

\section{Acknowledgements}

This study was supported by National Nature Science Foundation of China (No. 30571701; No. 30600541)

\section{Author details \\ ${ }^{1}$ Central Laboratory, First Affiliated Hospital of China Medical University, Shenyang 110001, China. ${ }^{2}$ Department of Immunology, College of Basic Medical Sciences, China Medical University, Shenyang 110001, China. ${ }^{3}$ Department of Biotherapy, Fourth Affiliated Hospital of China Medical University, Shenyang 110032, China. ${ }^{4}$ Department of Dermatology, First Affiliated Hospital of China Medical University, Shenyang 110001, China.}

Received: 9 May 2012 Accepted: 2 November 2012

Published: 8 November 2012

\section{References}

1. Gordon J, Manley NR: Mechanisms of thymus organogenesis and morphogenesis. Development 2011, 138:3865-3878.

2. Bleul CC, Boehm T: BMP signaling is required for normal thymus development. J Immunol 2005, 175:5213-5221.

3. Scott DK, Lord R, Muller HK, Malley RC, Woods GM: Proteomics identifies enhanced expression of stefin A in neonatal murine skin compared with adults: functional implications. Br J Dermatol 2007, 156:1156-1162.

4. Carrette O, Burkhard PR, Hochstrasser DF, Sanchez JC: Age-related proteome analysis of the mouse brain: a 2-DE study. Proteomics 2006, 6:4940-4949.

5. Pascual A, Romero-Ruiz A, Lopez-Barneo J: Differential proteomic analysis of adrenal gland during postnatal development. Proteomics 2009, 9:2946-2954.

6. Kawakami T, Nagata T, Muraguchi A, Nishimura T: Proteomic approach to apoptotic thymus maturation. J Chromatogr B Analyt Technol Biomed Life Sci 2003, 787:223-229.

7. Shiraishi J, Utsuyama M, Seki S, Akamatsu H, Sunamori M, Kasai M, Hirokawa K: Essential microenvironment for thymopoiesis is preserved in human adult and aged thymus. Clin Dev Immunol 2003, 10:53-59.

8. Tumani H, Lehmensiek V, Lehnert S, Otto M, Brettschneider J: 2D DIGE of the cerebrospinal fluid proteome in neurological diseases. Expert Rev Proteomics 2010, 7:29-38.

9. Jensen $L$, Kuhn M, Stark M, Chaffron S, Creevey C, Muller J, Doerks T, Julien P, Roth A, Simonovic M, et al: STRING 8-a global view on proteins and their functional interactions in 630 organisms. Nucleic Acids Res 2009, 37:D412-D416.

10. Campostrini N, Areces LB, Rappsilber J, Pietrogrande MC, Dondi F, Pastorino F, Ponzoni M, Righetti PG: Spot overlapping in two-dimensional maps: a serious problem ignored for much too long. Proteomics 2005, 5:2385-2395.

11. Hunsucker SW, Duncan MW: Is protein overlap in two-dimensional gels a serious practical problem? Proteomics 2006, 6:1374-1375.
12. Swatton JE, Prabakaran S, Karp NA, Lilley KS, Bahn S: Protein profiling of human postmortem brain using 2-dimensional fluorescence difference gel electrophoresis (2-D DIGE). Mol Psychiatry 2004, 9:128-143.

13. Yu HR, Kuo HC, Huang HC, Chen TY, Huang LT, Tain YL, Chen CC, Sheen JM, Lin IC, Ou CY, et al: Identification of immunodeficient molecules in neonatal mononuclear cells by proteomic differential displays. Proteomics 2011, 11:3491-3500.

14. Tondeleir D, Vandamme D, Vandekerckhove J, Ampe C, Lambrechts A: Actin isoform expression patterns during mammalian development and in pathology: insights from mouse models. Cell Motil Cytoskeleton 2009, 66:798-815.

15. Bunnell TM, Burbach BJ, Shimizu Y, Ervasti JM: beta-Actin specifically controls cell growth, migration, and the G-actin pool. Mol Biol Cell 2011, 22:4047-4058.

16. Rubenstein PA: The functional importance of multiple actin isoforms. Bioessays 1990, 12:309-315.

17. Shawlot W, Deng JM, Fohn LE, Behringer RR: Restricted beta-galactosidase expression of a hygromycin-lacZ gene targeted to the beta-actin locus and embryonic lethality of beta-actin mutant mice. Transgenic Res 1998, 7:95-103.

18. Menez J, Le Maux Chansac B, Dorothee G, Vergnon I, Jalil A, Carlier MF, Chouaib S, Mami-Chouaib F: Mutant alpha-actinin-4 promotes tumorigenicity and regulates cell motility of a human lung carcinoma. Oncogene 2004, 23:2630-2639.

19. Tooley AJ, Gilden J, Jacobelli J, Beemiller P, Trimble WS, Kinoshita M, Krummel MF: Amoeboid T lymphocytes require the septin cytoskeleton for cortical integrity and persistent motility. Nat Cell Biol 2009, 11:17-26.

20. Beemiller P, Krummel MF: Mediation of T-cell activation by actin meshworks. Cold Spring Harb Perspect Biol 2010, 2:a002444.

21. Lasserre R, Alcover A: Cytoskeletal cross-talk in the control of T cell antigen receptor signaling. FEBS Lett 2010, 584:4845-4850.

22. Ma M, Zhou L, Guo X, Lv Z, Yu Y, Ding C, Zhang P, Bi Y, Xie J, Wang L, et al: Decreased cofilin1 expression is important for compaction during early mouse embryo development. Biochim Biophys Acta 2009, 1793:1804-1810.

23. Jin EH, Shim SC, Kim HG, Chae SC, Chung HT: Polymorphisms of COTL1 gene identified by proteomic approach and their association with autoimmune disorders. Exp Mol Med 2009, 41:354-361.

24. Dai H, Huang W, Xu J, Yao B, Xiong S, Ding H, Tang Y, Liu H, Wu J, Shi Y Binding model of human coactosin-like protein with filament actin revealed by mutagenesis. Biochim Biophys Acta 2006, 1764:1688-1700.

25. Lappalainen P, Kessels MM, Cope MJ, Drubin DG: The ADF homology (ADF-H) domain: a highly exploited actin-binding module. Mol Biol Cell 1998, 9:1951-1959.

26. Christie DA, Lemke CD, Elias IM, Chau LA, Kirchhof MG, Li B, Ball EH, Dunn SD, Hatch GM, Madrenas J: Stomatin-like protein 2 binds cardiolipin and regulates mitochondrial biogenesis and function. Mol Cell Biol 2011, 31:3845-3856

27. Kirchhof MG, Chau LA, Lemke CD, Vardhana S, Darlington PJ, Marquez ME, Taylor R, Rizkalla K, Blanca I, Dustin ML, Madrenas J: Modulation of T cell activation by stomatin-like protein 2. J Immunol 2008, 181:1927-1936.

28. Beachy SH, Kisailus AJ, Repasky EA, Subjeck JR, Wang XY, Kazim AL: Engineering secretable forms of chaperones for immune modulation and vaccine development. Methods 2007, 43:184-193.

29. Neuer A, Spandorfer SD, Giraldo P, Jeremias J, Dieterle S, Korneeva I, Liu HC, Rosenwaks Z, Witkin SS: Heat shock protein expression during gametogenesis and embryogenesis. Infect Dis Obstet Gynecol 1999, 7:10-16.

30. Christensen JH, Nielsen MN, Hansen J, Fuchtbauer A, Fuchtbauer EM, West $\mathrm{M}$, Corydon TJ, Gregersen N, Bross P: Inactivation of the hereditary spastic paraplegia-associated Hspd1 gene encoding the Hsp60 chaperone results in early embryonic lethality in mice. Cell Stress Chaperones 2010, 15:851-863.

31. Setiady YY, Agersborg S, Samy ET, Lewis JE, Tung KS: Neonatal autoimmune disease: influence of CD4+ CD25+ regulatory T cells. Int Rev Immunol 2005, 24:227-245.

32. Li J, Sejas DP, Rani R, Koretsky T, Bagby GC, Pang Q: Nucleophosmin regulates cell cycle progression and stress response in hematopoietic stem/progenitor cells. J Biol Chem 2006, 281:16536-16545.

33. Frehlick LJ, Eirin-Lopez JM, Ausio J: New insights into the nucleophosmin/ nucleoplasmin family of nuclear chaperones. Bioessays 2007, 29:49-59. 
34. Grisendi S, Bernardi R, Rossi M, Cheng K, Khandker L, Manova K, Pandolfi PP: Role of nucleophosmin in embryonic development and tumorigenesis. Nature 2005, 437:147-153.

35. Ocklind G, Talts J, Fassler R, Mattsson A, Ekblom P: Expression of tenascin in developing and adult mouse lymphoid organs. J Histochem Cytochem 1993, 41:1163-1169.

36. Freitas CS, Lyra JS, Dalmau SR, Savino W: In vivo and in vitro expression of tenascin by human thymic microenvironmental cells. Dev Immunol 1995, 4:139-147.

37. Clark RA, Erickson HP, Springer TA: Tenascin supports lymphocyte rolling. J Cell Biol 1997, 137:755-765.

38. Adkins B, Leclerc C, Marshall-Clarke S: Neonatal adaptive immunity comes of age. Nat Rev Immunol 2004, 4:553-564.

39. Opiela SJ, Koru-Sengul T, Adkins B: Murine neonatal recent thymic emigrants are phenotypically and functionally distinct from adult recent thymic emigrants. Blood 2009, 113:5635-5643.

40. Sijts EJ, Kloetzel PM: The role of the proteasome in the generation of MHC class I ligands and immune responses. Cell Mol Life Sci 2011, 68:1491-1502.

41. Melnikova VI, Sharova NP, Maslova EV, Voronova SN, Zakharova LA: Ontogenesis of rat immune system: Proteasome expression in different cell populations of the developing thymus. Cell Immunol 2010, 266:83-89.

42. Tonomura N, McLaughlin K, Grimm L, Goldsby RA, Osborne BA: Glucocorticoid-induced apoptosis of thymocytes: requirement of proteasome-dependent mitochondrial activity. J Immunol 2003, 170:2469-2478

43. Garcia AM, Fadel SA, Cao S, Sarzotti M: T cell immunity in neonates. Immunol Res 2000, 22:177-190.

44. Cao S, Carlesso G, Osipovich AB, Llanes J, Lin Q, Hoek KL, Khan WN, Ruley HE: Subunit 1 of the prefoldin chaperone complex is required for lymphocyte development and function. J Immunol 2008, 181:476-484.

doi:10.1186/1477-5956-10-65

Cite this article as: Cai et al.: Proteomics identifies differentially expressed proteins in neonatal murine thymus compared with adults. Proteome Science 2012 10:65.

\section{Submit your next manuscript to BioMed Central and take full advantage of:}

- Convenient online submission

- Thorough peer review

- No space constraints or color figure charges

- Immediate publication on acceptance

- Inclusion in PubMed, CAS, Scopus and Google Scholar

- Research which is freely available for redistribution 